\title{
Integrierte Versorgung in der Schweiz
}

\author{
Séverine Schusselé Filliettaz ${ }^{a}$, Peter Berchtold ${ }^{b}$, Dimitri Kohler ${ }^{c}$, Isabelle Peytremann-Bridevaux ${ }^{d}$ \\ ${ }^{a}$ Forum Managed Care; Institut de médecine sociale et préventive, Lausanne; ${ }^{\mathrm{b}}$ Dr. med., Forum Managed Care, Mitglied FMH; ${ }^{\mathrm{c}}$ Schweizerisches Gesund- \\ heitsobservatorium (OBSAN); d Dr. med., Institut de médecine sociale et préventive, Lausanne, Mitglied FMH
}

Die Erkenntnis, dass eine qualitativ hochwertige Behandlung mehr Kommunikation, Koordination und Kooperation zwischen den Leistungserbringern verlangt, ist heute weitgehend anerkannt. Mit der ersten nationalen Erhebung zur Integrierten Versorgung in der Schweiz liegt ausserdem eine Fülle an Projekten und Erfahrungen vor. Wie lassen sich diese nutzen, um die Integration voranzubringen, und welche intelligenten Lösungsansätze lassen sich daraus ableiten?

Die Fragmentierung der Gesundheitsversorgung und die zunehmende Prävalenz chronischer Krankheiten setzen die Gesundheitssysteme unter Druck. Vor diesem Hintergrund sind in der Schweiz wie im Ausland in den letzten Jahren zahlreiche Initiativen entstanden, um die Patientenversorgung stärker zu koordinieren und integrieren. Der rasante Aufschwung dieser Entwicklungen hat das Forum Managed Care, das Institut universitaire de médecine sociale et préventive (Lausanne) und das Schweizerische Gesundheitsobservatorium (Obsan) dazu veranlasst, eine Studie durchzuführen [1]. Deren Ziel war es, eine detaillierte Übersicht aller Initiativen zur integrierten Gesundheitsversorgung in der Schweiz zu geben, diese sichtbar zu machen und den Erfahrungsaustausch zu fördern.

\section{Nationale Erhebung}

Die gesamtschweizerische Erhebung wurde zwischen Juli 2015 und Juli 2016 durchgeführt. Zur Teilnahme an der Erhebung wurden Initiativen eingeladen, die vier Kriterien erfüllen mussten: 1) Die Integrationsprinzipien sind formalisiert; 2) mindestens zwei Ebenen der Gesundheitsversorgung sind integriert/koordiniert; 3) mindestens zwei Gesundheitsfach- bzw. Berufsgruppen sind beteiligt und 4) die Initiativen sind im Zeitpunkt der Erhebung aktiv.

Um eine möglichst vollständige Erhebung zu gewährleisten, wurden alle wichtigen Akteure im Schweizer Gesundheitswesen auf nationaler, kantonaler und lokaler Ebene systematisch kontaktiert: Leistungserbringer,
Finanzierungsträger, Behörden, Ausbildungs- und Forschungseinrichtungen sowie Fach- und gemeindenahe Organisationen. Die Initiativen, die sich für eine Teilnahme an der Erhebung qualifizierten, erhielten anschliessend einen Link zu einem Online-Fragebogen mit Fragen zu Versorgungs-Kontext, Zielgruppen, beteiligten Fachpersonen, Leistungsangebot, integrierten Ebenen, Finanzierung und Evaluation.

Aus insgesamt 853 Kontaktnahmen qualifizierten sich 172 Initiativen für eine Teilnahme an der Erhebung. Dank einer ausgesprochen hohen Rücklaufquote von über 94\% konnten Daten zu 155 Hauptinitiativen und 7 Unterprogrammen ausgewertet werden. Dazu wurden die 155 Hauptinitiativen nachträglich einer Kategorie zugeordnet: «Spezifische Zielgruppen» (34\%), «Psychische Gesundheit \& Psychiatrie» (26\%), "Gesundheitszentren» (16\%), «Übergänge \& Koordination» (16\%), «Ärztenetzwerke» (6\%), «Medikamente» (5\%).

\footnotetext{
Nationales Symposium Integrierte Versorgung, 14. Juni 2017, Kursaal Bern

Vor ein paar Jahren noch bedeutete Integration in der Schweiz vor allem ärztliche Grundversorgernetze. Nach wie vor leisten diese einen wichtigen Beitrag zur Integrierten Versorgung. Gleichzeitig entstand eine Vielzahl an neuen Formen der Integration. Unter dem Titel «Smarter Healthcare - die Kunst der klugen Integration" werden am Nationalen Symposium 2017 des fmc Schweizer Forum für Integrierte Versorgung künftige Entwicklungen, Strategien und Vernetzungspraktiken präsentiert und diskutiert. Ausserdem können die Teilnehmenden das Thema in moderierten Tischgesprächen und Workshops vertiefen und ihre Expertise einbringen. Weitere Informationen zum fmc-Symposium 2017 finden Sie auf www.fmc.ch.
} 


\section{Eindrückliche Entwicklung und Vielfalt}

Die Integrierte Versorgung in der Schweiz hat sich in den letzten Jahren eindrücklich beschleunigt: Während die erste Hälfte der 162 Integrationsinitiativen in der Zeit von 1990 bis 2009 entstand, wurden die zweiten 50\% in den Jahren 2010 bis 2016 lanciert. Bis 2012 war die Mehrheit der Initiativen in der deutschsprachigen Schweiz anzutreffen, heute finden wir dieses Verhältnis umgekehrt: $52 \%$ sind in der lateinischen Schweiz, 45\% in der deutschen Schweiz und 3\% in den beiden Regionen aktiv. Zwischen den Sprachregionen bestehen relevante Unterschiede: In der Deutschschweiz gibt es mehr «Ärztenetzwerke» und «Gesundheitszentren", in der lateinischen Schweiz hingegen sind Initiativen häufiger, die auf «spezifische Zielgruppen», auf «Medikamente» und auf die «Koordination» bestimmter Übergänge ausgerichtet sind.

Eindrücklich sind auch die unterschiedlichen Entwicklungen in den genannten Kategorien (Abb. 1). Die stärkste Zunahme finden wir in den beiden Kategorien «Spezifische Zielgruppen» und «Psychische Gesundheit \& Psychiatrie». Das ist insofern beachtenswert, als dass in diesen beiden Kategorien bzw. in der Betreuung von chronisch und psychisch kranken Patienten ein

CH-6314 Neuägeri besonders hoher Koordinations- und Integrationsbedarf besteht. Auffallend ist auch die rasche Zunahme in der nicht-krankheitsspezifischen Kategorie «Übergänge \& Koordination».

Die Integrationsinitiativen zeichnen sich durch eine grosse, wenn auch unterschiedliche Breite an involvierten Fachpersonen aus: Im Durchschnitt sind rund fünf Gesundheitsfach- bzw. Berufsgruppen beteiligt. Das grösste Spektrum fanden wir in den «Gesundheitszentren" mit sieben Berufsgruppen, gefolgt von den «Spezifischen Zielgruppen» mit durchschnittlich sechs und den übrigen Kategorien mit durchschnittlich drei bis vier Berufsgruppen. Zwischen den Kategorien fanden wir weitere Unterschiede. So integrieren und koordinieren Gesundheitszentren die meisten Versorgungsebenen, während die medikamentenbezogenen Projekte diesbezüglich am engsten gefasst sind.

\section{Fazit}

Diese nationale Erhebung zur Integrierten Versorgung erfasst erstmals systematisch Initiativen der Integrierten Versorgung bei allen wichtigen Akteuren des Schweizer Gesundheitswesens. Wesentlichstes Ergebnis ist sicher, dass wir in allen Regionen der Schweiz eine eindrückliche und breit gefächerte Entwicklung der Initiativen zu Integrierter Versorgung beobachten können. Angesichts unseres föderalistischen Systems, dem wir gerne eine Mitschuld an der Fragmentierung der Gesundheitsversorgung zuschreiben, und angesichts der bekannterweise bescheidenen Anreize für Integrierte Versorgung sind diese Ergebnisse ausgesprochen bemerkenswert. Und sie spiegeln die Stärken dieser Entwicklungen: Es entstehen differenzierte, auf spezifische Bedürfnisse von Regionen oder Zielgruppen ausgerichtete Betreuungsangebote.

\section{Bildnachweis}

@) Séverine Schusselé Filliettaz

\section{Referenz}

1 Schusselé Filliettaz S, Kohler D, Berchtold P, Peytremann-Bridevaux I (2017). Soins intégrés en Suisse. Résultats de la $1^{\text {re }}$ enquête (2015-2016) (Obsan Dossier 57). Neuchâtel: Observatoire suisse de la santé. www.obsan.ch

Abbildung 1: Kumulative Anzahl der Initiativen zu Integrierter Versorgung pro Kategorie 1990-2016. 